\title{
Feasibility and Efficacy of Concurrent Chemoradiotherapy in Elderly Patients with Esophageal Squamous Cell Carcinoma: a Respective Study of 116 Cases from a Single Institution
}

\author{
Xue $\mathrm{Li}^{\circledR}$, Lu-Jun Zhao ${ }^{\star}$, Ning-Bo Liu ${ }^{1}$, Wen-Cheng Zhang, Qing-Song Pang, \\ Ping Wang*, Zhi-Yong Yuan
}

\begin{abstract}
Background: To evaluate the safety and efficacy of combined chemoradiotherapy or radiotherapy alone in elderly patients with esophageal carcinoma to identify the best method of treatment. Materials and Methods: One hundred and sixteen patients with esophageal carcinoma aged 70 and older who received definitive radiotherapy or chemoradiotherapy entered the study. Overall survival (OS), disease-free survival (DFS) and treatmentrelated toxicities were assessed. Results: The median OS of the overall population was 17.9 months. For patients treated with CCRT, SCRT and radiotherapy alone, the median OS was 22.3 months, 18.0 months and 12.4 months respectively $(P=0.044)$. Median $\mathrm{OS}$ for patients treated with radiotherapy dose $\geq 60 \mathrm{~Gy}$ and $<60 \mathrm{~Gy}$ was 20.2 months and 10.9 months respectively $(p=0.017)$. By univariate analysis, Chemoradiotherapy (include CCRT and sCRT) and radiotherapy dose $\geq 60 \mathrm{~Gy}$ were found to achieve higher survival rates compared with radiotherapy alone and radiotherapy dose $<60 \mathrm{~Gy}(P=0.015, P=0.017)$. By multivariate analysis, chemoradiotherapy $(H R=1.645$, $P=0.022)$ and radiotherapy dose $\geq 60 G y(H R=1.642, P=0.025)$ were identified as independent prognostic factors of OS. Conclusions: Definitive concurrent chemoradiotherapy could be considered as a feasible and effective treatment in esophageal carcinoma patients aged 70 and older. Radiotherapy dose $60 \mathrm{~Gy}$ is an effective treatment option compared with standard dose radiotherapy, while higher doses are not beneficial to improve survival.
\end{abstract}

Keywords: Esophageal squamous cell carcinoma - elderly - concurrent chemoradiotherapy - survival - toxicity

Asian Pac J Cancer Prev, 16 (4), 1463-1469

\section{Introduction}

Esophageal carcinoma (EC) is the eighth most common cancer and the sixth most common cause of cancer death worldwide (Chen et al., 2011). In China, the morbidity of EC ranks sixth and is the fourth leading cause of cancer-related mortality (Liu et al., 2013; Yuan et al., 2014). Squamous cell carcinoma (SCC) is the predominant histological type of EC worldwide, which accounts for $>90 \%$ of all types of EC in China (Zhang, 2013). For different types of EC, the risk increases with age, with a mean age at diagnosis of 67 years, and the incidence of SCC peaks in the seventh decade of life (Igissinov et al., 2011). Therefore, the control of EC is an urgent issue, especially in elderly patients.

Esophagectomy remains the primary treatment for early stage EC, and a multimodal treatment with a neoadjuvant chemotherapy or a combined chemoradiotherapy followed by surgery or definitive chemoradiotherapy is recommended for locally advanced cancers (Mirinezhad et al., 2013; D’ Journo et al., 2014). However, EC patients aged $>70$ years old are under-represented in most randomized trials and there are lack of evidence-based strategies for the elderly population with EC. Therefore, the current retrospective study was designed to evaluate the safety and efficacy of combined chemoradiotherapy or radiotherapy alone in elderly patients with EC to identify the best method of treatment.

\section{Materials and Methods}

\section{Patients'data}

Patients' inclusion criteria included: 1) aged 70 and older, 2) histologically confirmed esophageal squamous cell carcinoma, 3) Karnofsky Performance Status (KPS) of at least 70,4) no distinct comorbidity such as severe pulmonary disease or cardiovascular disease, with the attending physician assuring the medical status of the patient other than age, 5) had not undergone surgical resection, 6) patients or family members were contactable and clear survival status. This retrospective study was approved by our Institutional Review Board. 
Tumor evaluation was based on esophagoscopy, barium esophagography, CT scan (chest and abdominal) and ultrasonography (neck and abdominal). Esophageal ultrasonography was not used routinely, therefore, it was difficult to classify T stage by CT scan only. TNM staging was defined according to the American Joint Committee on Cancer (AJCC) criteria, $6^{\text {th }}$ edition. Physician-reported hematological, esophageal and pulmonary toxicities of all eligible patients were evaluated according to the common toxicity criteria for adverse events version 3.0 (CTCAE v3.0). The most relevant treatment modalities in elderly patients remain a subject of debate and patients' treatment modality was decided on patients' choose and doctor's clinical experiments.

\section{Radiotherapy}

CT-based radiation planning and 3D conformal radiotherapy (3D-CRT) or intensity modulated radiotherapy (IMRT) were used in the patients. All patients were treated by conventional fractionation (1.8-2Gy per fraction, one fraction per day and five fractions per week). The median dose of radiation delivered was 60Gy (range 20-70Gy). The radiation parameters were as follows: 1) energy, 6 $\mathrm{MV}$; 2) The gross tumor volume (GTV), including primary tumor and involved lymph nodes, the criteria of lymph node positivity on the CT scan were: short axis size $\geq$ $10 \mathrm{~mm}$, lymph node with infiltrative margin, or central necrosis; 3) The clinical target volume (CTV) comprised GTV with 3 to $5 \mathrm{~cm}$ superior and inferior margins and 0.5 to $0.8 \mathrm{~cm}$ lateral margins and nodal risk regions. The nodal risk regions including supraclavicular, periesophageal and station 2, 4, 7 lymph nodes for upper one-third tumor, periesophageal and station 2, 4, 7 lymph nodes for middle one-third tumor, and periesophageal, station 4, 7 lymph nodes and left gastric for lower one-third tumor. Lymph node involvement descriptor were according to the MD-ATS map (Mountain et al., 1997); 4) The planning target volume (PTV), including a minimum of $0.5-1 \mathrm{~cm}$ surrounding the CTV.

\section{Chemotherapy}

Fifty-six patients received concomitant chemotherapy: 13 had weekly docetaxel $\left(40 \mathrm{mg} / \mathrm{m}^{2}\right)$ (Shen et al., 2012), 23 had cisplatin/5-FU (2 cycles with $5-\mathrm{FU} 400 \mathrm{mg} /$ $\left(\mathrm{m}^{2} . \mathrm{d}\right)$ at days $1-5$ and cisplatin $20 \mathrm{mg} /\left(\mathrm{m}^{2} . \mathrm{d}\right)$ at days 1-3) (Mirinezhad et al., 2013), 8 had weekly carboplatin/ paclitaxel (4 courses with carboplatin $20 \mathrm{mg} /\left(\mathrm{m}^{2} . \mathrm{d}\right)$ and paclitaxel $75 \mathrm{mg} /\left(\mathrm{m}^{2} \cdot \mathrm{d}\right)$ at day 1$)$, and 12 had doxifluridine (200 mg/d).

\section{Statistical methods}

Overall survival (OS) was calculated from the date of chemoradiotherapy or radiotherapy initiation until the date of death or the date of last follow-up. Progression-free survival (PFS) was calculated from the date of chemoradiotherapy or radiotherapy initiation to the date of documented failure (local recurrence or metastasis occurrence) or the date of the last follow-up for those remaining. Survival curves were determined using the Kaplan-Meier method. Prognostic factors for OS and PFS were obtained using the log-rank test and statistical significance was defined with a $p$-value $<0.05$. All prognostic factors with a $p<0.2$ were included for a multivariate analysis using a Cox regression. Statistical analyses were performed using SPSS 17.0 (SPSS, Inc., Chicago, IL, USA).

Table 1. Patients and Tumor Characteristics

\begin{tabular}{|c|c|c|c|c|c|c|c|c|c|}
\hline \multirow[t]{2}{*}{ Characteristic } & \multirow{2}{*}{$\begin{array}{c}\text { Total } \\
\text { No. Patients }(\%)\end{array}$} & \multicolumn{3}{|c|}{ Chemotherapy } & \multirow[b]{2}{*}{$P$ value } & \multicolumn{4}{|c|}{ Radiotherapy dose } \\
\hline & & cCRT & sCRT & eRT & & $<60 \mathrm{~Gy}$ & $60 \mathrm{~Gy}$ & $<60 \mathrm{~Gy}$ & $P$ value \\
\hline Gender & & & & & 0.294 & & & & 0.133 \\
\hline Male & $84(72.4)$ & $26(81.8)$ & $15(62.5)$ & $43(71.7)$ & & $24(72.7)$ & $27(62.8)$ & $33(82.5)$ & \\
\hline Female & $32(27.6)$ & $6(18.3)$ & $9(37.5)$ & $17(28.3)$ & & $9(27.3)$ & $16(37.2)$ & $7(17.5)$ & \\
\hline Median age(range) & $76(70-90)$ & $74(70-90) 7$ & $74(70-88) 78$ & $8(87-70)$ & & $76(70-90) 76$ & $(89-70)$ & $76.5(70-88)$ & \\
\hline Complication & & & & & 0.153 & & & & 0.48 \\
\hline Yes & $62(52.4)$ & $15(46.9)$ & $17(70.8)$ & $30(50)$ & & $17(51.5)$ & $26(60.5)$ & $19(47.5)$ & \\
\hline No & $54(46.6)$ & $17(53.1)$ & $7(29.2)$ & $30(50)$ & & $16(48.5)$ & $17(39.5)$ & $21(52.5)$ & \\
\hline Stage & & & & & 0.487 & & & & 0.773 \\
\hline I-IIa & $22(19)$ & $8(25)$ & $4(16.7)$ & $10(16.7)$ & & $5(15.2)$ & $7(16.3)$ & $10(25)$ & \\
\hline IIb-III & $44(37.9)$ & $10(31.3)$ & $7(29.2)$ & $27(45)$ & & $12(36.4)$ & $18(41.9)$ & $14(35)$ & \\
\hline IV & $50(43.1)$ & $14(43.8)$ & $13(54.2)$ & $23(38.3)$ & & $16(48.5)$ & $18(41.9)$ & $16(40)$ & \\
\hline Tumor location & & & & 0.71 & & & & 0.198 & \\
\hline Upper one-third & $42(36.2)$ & $14(43.8)$ & $7(29.2)$ & $21(35)$ & & $15(45.5)$ & $16(37.2)$ & $11(27.5)$ & \\
\hline Middle one-third & $58(50)$ & $15(46.9)$ & $14(58.3)$ & $29(48.3)$ & & $14(42.2)$ & $24(55.8)$ & $20(50)$ & \\
\hline Lower one-third & $16(13.8)$ & $3(9.4)$ & $3(12.5)$ & $10(16.7)$ & & $4(12.1)$ & $3(7)$ & $9(22.5)$ & \\
\hline Radiotherapy & & & & & 0.002 & & & & 0.407 \\
\hline 3D-CRT & $73(62.9)$ & $12(37.5)$ & $19(79.2)$ & $42(70)$ & & $21(63.6)$ & $24(55.8)$ & $28(70)$ & \\
\hline IMRT & $43(37.1)$ & $20(62.5)$ & $5(20.8)$ & $18(30)$ & & $12(36.4)$ & $19(44.2)$ & $12(30)$ & \\
\hline Chemotherapy & & & & & & & & & 0.595 \\
\hline cCRT & $32(27.6)$ & & & & & $12(36.4)$ & $11(25.6)$ & $9(22.5)$ & \\
\hline sCRT & $24(20.7)$ & & & & & $7(21.2)$ & $10(23.3)$ & $7(17.5)$ & \\
\hline eRT & $60(51.7)$ & & & & & $14(42.4)$ & $22(51.2)$ & $24(60)$ & \\
\hline
\end{tabular}

*cCRT, current chemoradiotherapy; sCRT, sequential chemoradiotherapy; eRT, exclusive radiotherapy; 3D-CRT, 3D conformal radiotherapy; IMRT, intensity modulated radiotherapy 
DOI:http://dx.doi.org/10.7314/APJCP.2015.16.4.1463

Feasibility and Efficacy of Concurrent Chemoradiotherapy in Elderly Patients with Esophageal SCC

Table 2. Univariate Results

\begin{tabular}{|c|c|c|c|c|c|c|c|c|}
\hline Variable & & Number & Median OS & $\mathrm{x}^{2}$ & $P$ value & Median PFS & $\mathrm{x}^{2}$ & $P$ value \\
\hline \multirow[t]{2}{*}{ Gender } & Male & 84 & 12.4 & 7.407 & 0.006 & 15.2 & 8.26 & 0.004 \\
\hline & Female & 32 & 24.9 & & & 24.9 & & \\
\hline \multirow[t]{2}{*}{ N Stage } & No & 32 & 24.7 & 1.357 & 0.244 & 19.7 & 0.846 & 0.358 \\
\hline & N1 & 84 & 12.7 & & & 15.3 & & \\
\hline \multirow[t]{2}{*}{ M Stage } & M0 & 66 & 18.2 & 0.002 & 0.967 & 18.4 & 1.277 & 0.258 \\
\hline & M1 & 50 & 14.2 & & & 15.2 & & \\
\hline \multirow[t]{3}{*}{ Clinical Stage } & I-IIa & 22 & 21.4 & 0.893 & 0.64 & 17.8 & 1.296 & 0.523 \\
\hline & IIb-III & 44 & 17.3 & & & 24.7 & & \\
\hline & IV & 50 & 14.2 & & & 15.2 & & \\
\hline \multirow{2}{*}{ Radiotherapy } & 3D-CRT & 73 & 13.9 & 2.744 & 0.098 & 17.1 & 0.834 & 0.361 \\
\hline & IMRT & 43 & 22.3 & & & 18.7 & & \\
\hline \multirow[t]{2}{*}{ Radiation dose } & $\geq 60 \mathrm{~Gy}$ & 76 & 20.2 & 5.68 & 0.017 & 22.3 & 8.542 & 0.003 \\
\hline & $<60 \mathrm{~Gy}$ & 40 & 10.9 & & & 12.4 & & \\
\hline \multirow[t]{2}{*}{ Treatment modality } & cCRT and sCRT & 56 & 19 & 5.859 & 0.015 & 18.2 & 1.756 & 0.185 \\
\hline & RT alone & 60 & 12.4 & & & 17.8 & & \\
\hline \multirow{2}{*}{ Chemotherapy } & $5-\mathrm{Fu}$ & 17 & 37.7 & 2.656 & 0.103 & 18.2 & 0.084 & 0.772 \\
\hline & Others & 16 & 17.3 & & & 18.7 & & \\
\hline
\end{tabular}

*CCRT=concurrent chemoradiotherapy, sCRT=sequential chemoradiotherapy, 3D-CRT=three-dimensional conformal radiotherapy, IMRT=intensitymodulated radiotherapy, OS=overall survival, $\mathrm{PFS}=$ progression-free surviva

Table 3. Multivariate Results (OS)

\begin{tabular}{|c|c|c|c|c|c|}
\hline Variable & $\beta$ value & SE value & $x^{2}$ & $P$ value & $\operatorname{Exp}(B)$ \\
\hline Gender (Male, Female) & -0.801 & 0.268 & 8.967 & 0.003 & 0.449 \\
\hline $\mathrm{N}$ stage $(\mathrm{N} 0, \mathrm{~N} 1)$ & 0.472 & 0.362 & 1.693 & 0.193 & 1.602 \\
\hline M stage (M0, M1) & 0.069 & 0.581 & 0.014 & 0.906 & 1.071 \\
\hline Clinical stage (I-IIa, IIb-III, IV) & -0.028 & 0.45 & 0.004 & 0.951 & 0.973 \\
\hline Radiotherapy (3D-CRT, IMRT) & -0.318 & 0.235 & 1.832 & 0.176 & 0.728 \\
\hline Radiation dose ( $\geq 60 \mathrm{~Gy},<60 \mathrm{~Gy})$ & 0.234 & 0.115 & 4.139 & 0.042 & 1.264 \\
\hline Treatment modality (cCRT and sCRT, RT alone) & 0.485 & 0.233 & 4.349 & 0.037 & 1.625 \\
\hline
\end{tabular}

*cCRT=concurrent chemoradiotherapy, sCRT=sequential chemoradiotherapy, 3D-CRT=three-dimensional conformal radiotherapy, IMRT=intensitymodulated radiotherapy

\section{Results}

\section{Patient and tumor characteristics}

Between January 2008 and January 2013, 1059 patients with EC underwent radiotherapy at the Department of Thoracic Oncology (Tianjin Cancer Hospital, Tianjin China) and 116 patients who met the inclusion criteria were included in the present study. Individuals were followed up until April 2014. The median follow-up period was 16.97 months (range, 1.77-71.56 months). Patient and tumor pretreatment characteristics are listed in Table 1 . There was no statistically significant difference in background factors between patients grouped according to treatment modality and radiotherapy dose. The median age of the patients was 76 years old (range, 70-90 years old). The prevalence of patients with comorbidities was $44.83 \%$ (52/116), including 15 patients $(12.9 \%)$ had chronic obstructive pulmonary disease, 17 patients $(14.7 \%)$ had diabetes, and 20 patients (17.2\%) had peripheral vascular disease. None of the patients had grade 3 or 4 cardiac dysfunction (according to the NYHA grading).

\section{Treatment characteristics}

Treatment characteristics are listed in table 2 . Thirtytwo patients received cCRT, 24 patients received sCRT and 60 patients received radiotherapy alone; 60 patients received RT dose $>60 \mathrm{~Gy}, 43$ patients received $60 \mathrm{~Gy}$ and 40 patients received $<60 \mathrm{~Gy}$. Dose reduction for toxicity was performed in 31 patients (26.7\%), including cCRT 11 patients (34.4\%), sCRT 6 patients (25\%) and radiotherapy alone 14 patients (23.3\%); radiotherapy dose $>60 \mathrm{~Gy} 12$ patients (33.3\%), 60Gy 12 patients (30.2\%) and <60Gy 9 patients (22.5\%). Treatment breaks from radiotherapy were required in $29.3 \%$ (34/116) of patients due to intolerance to acute radiotherapy reactions, including cCRT 13 patients (40.6\%), sCRT 7 patients (29.2\%) and radiotherapy alone 14 patients $(23.3 \%)$; radiotherapy dose $>60$ Gy 12 patients (36.4\%), 60Gy 14 patients $(32.6 \%)$ and $<60 \mathrm{~Gy} 9$ patients $(22.5 \%)$. The median duration was 3 days (range 1-10). A chemotherapy delay of more than 1 week was required in 14 patients treated with cCRT (43.8\%). Finally, the completion rate of the planned treatment was $56.3 \%$ (18/32), 58.3\% (14/24) and $65 \%(39 / 60)$ for patients treated with cCRT, sCRT and radiotherapy alone. One patient prescribed radiation dose 60Gy performed dose reduction for acute toxicity when radiation to $48 \mathrm{~Gy}$ and grouped to RT dose $<60 \mathrm{~Gy}$.

\section{Acute and late toxicity}

Acute toxicity grades 3 - 4 were observed in $17.2 \%$ (20/116) of patients. No patients suffered from acute grade 4 neutropenia and neutropenic fever during treatment. Acute grade 3 - 4 esophagitis was identified in $25 \%(8 / 32)$ of the cCRT patients, $16.7 \%(4 / 24)$ of the sCRT patients and $13.3 \%(8 / 60)$ of the radiotherapy alone patients, the difference between those groups were 


\begin{tabular}{lccccc}
\hline Variable & $\beta$ value & SE value & $\mathrm{x}^{2}$ & P value & Exp(B) \\
\hline Gender (Male, Female) & -0.863 & 0.285 & 9.153 & 0.002 & 0.422 \\
N stage (N0, N1) & 0.593 & 0.407 & 2.12 & 0.145 & 1.81 \\
M stage (M0, M1) & 0.78 & 0.644 & 1.468 & 0.226 & 2.181 \\
Clinical stage (I-IIa, IIb-III, IV) & -0.379 & 0.498 & 0.58 & 0.446 & 0.684 \\
Radiotherapy (3D-CRT,IMRT) & -0.252 & 0.248 & 1.036 & 0.309 & 0.777 \\
Radiation dose ( $\geq$ 60Gy, <60Gy) & 0.313 & 0.125 & 6.303 & 0.012 & 1.367 \\
Treatment modality (cCRT and sCRT, RT alone) & 0.379 & 0.244 & 2.4 & 0.121 & 1.46 \\
\hline
\end{tabular}

$*_{\mathrm{cCRT}}=$ concurrent chemoradiotherapy, $\mathrm{sCRT}=$ sequential chemoradiotherapy, $3 \mathrm{D}-\mathrm{CRT}=$ three-dimensional conformal radiotherapy, IMRT=intensitymodulated radiotherapy

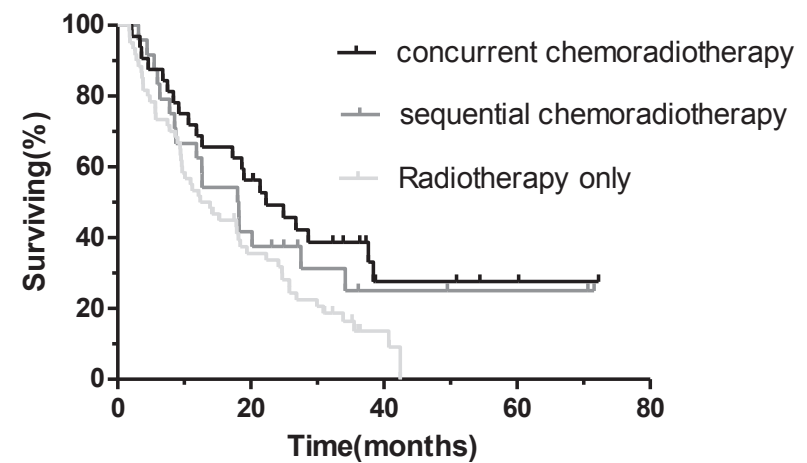

Figure 1. Overall Survival in Concurrent Chemoradiotherapy, Sequential Chemoradiotherapy and Radiotherapy Alone. The median overall survival was 22.3 months, 18 months and 12.4 months respectively $\left(\chi^{2}=6.266\right.$, $p=0.044)$

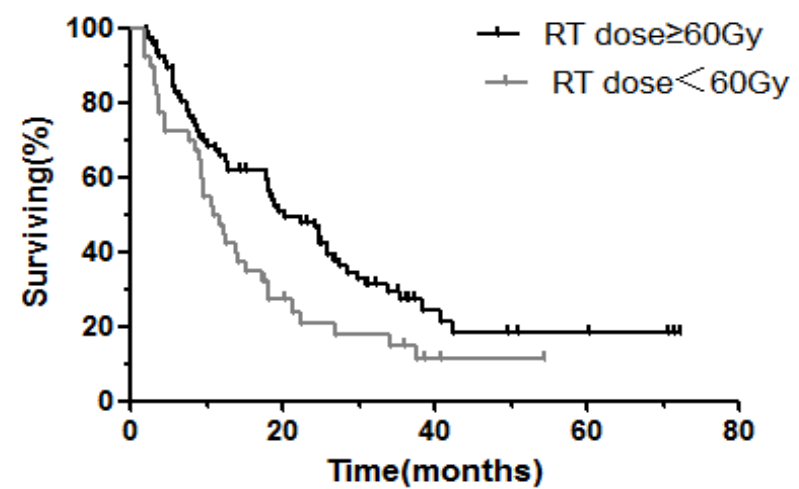

Figure 2. Overall Survival in Radiation Dose $\geq 60 G y$ and $<60 G y$. The median overall survival was 20.2 months in radiation dose $\geq 60 \mathrm{~Gy}$ as compared with 10.9 months in $<60 \mathrm{~Gy}$ $(p=0.0172)$

not statistically significant $\left(\mathrm{x}^{2}=1.911, p=0.385\right)$, and the difference on acute esophagitis between patients treated by radiotherapy dose $>60 \mathrm{~Gy}, 60 \mathrm{~Gy}$ and $<60 \mathrm{~Gy}$ were not statistically significant $\left(\mathrm{x}^{2}=1.792, p=0.408\right)$, with grade 3 - 4 esophagitis $24.2 \%(8 / 33), 16.3 \%(7 / 43)$ and $12.5 \%$ (5/40) respectively. No patients suffered from grade 3 - 4 pneumonitis. There was no late pulmonary toxicity. There were two treatment-related death $(4 \%)$ in the radiotherapy alone group. Of these two treatment-related deaths, one patient (radiation dose $41.4 \mathrm{~Gy} / 23 \mathrm{f}, 1.8 \mathrm{~Gy} / \mathrm{f}$ ) was due to esophageal bleeding during radiotherapy, and one (radiation dose 50.4Gy/28f, $1.8 \mathrm{~Gy} / \mathrm{f}$ ) due to tracheoesophageal fistula at 6.3 months after the initiation of treatment.

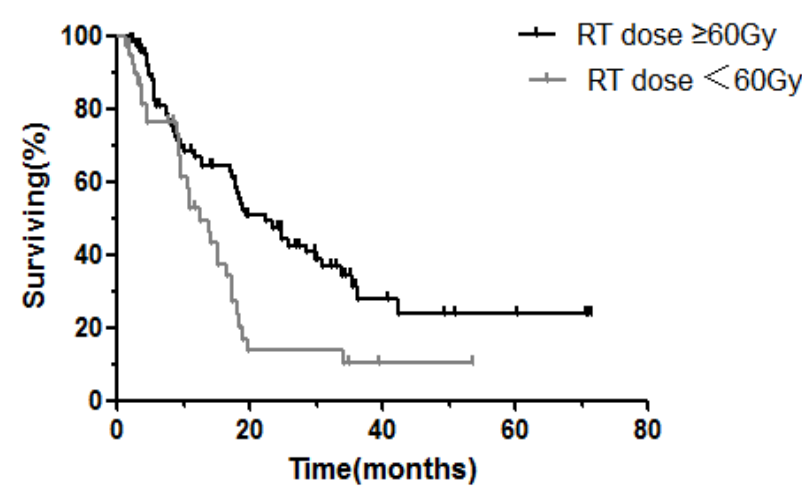

Figure 3. Progression-free survival in radiation dose $\geq 60 G y$ and $<60 G y$. The median progression-free survival was 22.3 months in radiation dose $\geq 60 \mathrm{~Gy}$ as compared with 12.4 months in $<60 \mathrm{~Gy}(p=0.0035)$

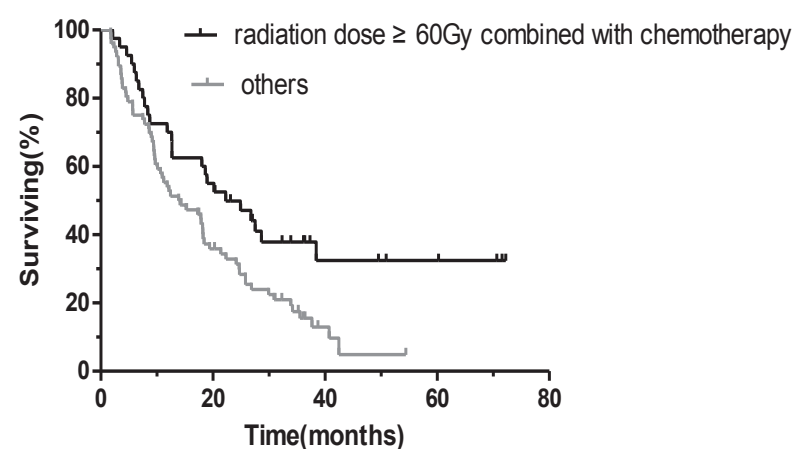

Figure 4. Overall Survival in Radiation Dose $\geq 60 G y$ Combined with Chemotherapy and Others. The median overall survival was 22.3 months, 13.9 months respectively $\left(\chi^{2}=6.769, p=0.009\right)$

\section{Treatment outcomes}

The median OS of the overall population was 17.9 months (95\%CI, 12.829-22.982), and 1, 2 and 3 yearsoverall survival rates were $59.1 \%, 38.4 \%$ and $23.2 \%$, respectively. The median PFS of the overall population was 17.9 months (95\%CI, 12.829-22.982), and 1, 2 and 3 years progression-free survival rates were $61.9 \%, 37.9 \%$, and $0 \%$, respectively. For patients treated with cCRT, sCRT and radiotherapy alone, the median OS was 22.3 months (95\%CI, 12.496-32.054), 18.0 months (95\%CI, 11.300-24.708) and 12.4 months (95\%CI, 7.007 - 17.831), respectively, the difference in OS between those groups was statistically significant $\left(\chi^{2}=6.266, p=0.044\right.$; Figure $1)$, while not different significantly in PFS between these groups $\left(\chi^{2}=1.761, p=0.414\right)$. The OS was significantly higher in patients treated with radiotherapy dose $\geq 60 \mathrm{~Gy}$ than that $<60 \mathrm{~Gy}\left(\chi^{2}=5.68, p=0.017\right.$; Figure 2$)$, with 


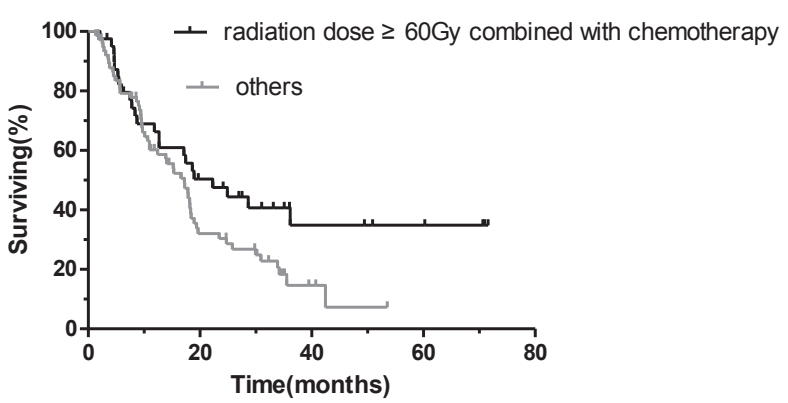

Figure 5. Progression-free Survival in Radiation Dose $\geq 60 G y$ Combined with Chemotherapy and Others. The median progression-free survival was 22.3 months, 17.2 months respectively $\left(\chi^{2}=3.953, p=0.047\right)$

median OS 20.2 months (95\%CI, 14.288-26.123) and 10.9 months (95\%CI, 7.173-14.708), respectively, and the difference in PFS between the two groups was statistically significant $\left(\chi^{2}=8.542, p=0.0035\right.$; Figure 3$)$, while the OS and PFS were not different significantly between $>60 \mathrm{~Gy}$ and 60Gy $\left(\chi^{2}=0.006, p=0.938 ; \chi^{2}=0.078, p=0.78\right)$. The OS and PFS were significantly higher in patients treated by radiation dose $\geq 60$ Gy combined with chemotherapy than others $\left(\chi^{2}=6.769, p=0.009\right.$, Figure $4 ; \chi^{2}=3.953, p=0.047$, Figure 5).

\section{Univariate and multivariate analyses}

By univariate analysis (table 2), patients treated with chemoradiotherapy (include cCRT and sCRT) were found to achieve higher survival rates compared with patients treated with radiotherapy alone, the difference between the two groups was statistically significant $(p=0.015)$; patients treated with radiotherapy dose $\geq 60 \mathrm{~Gy}$ achieved higher survival rates compared with patients treated with $<60 \mathrm{~Gy}$, the difference was statistically significant $(p=0.017)$. By multivariate analysis (table 3 , table 4 ), chemoradiotherapy (19.0 months if treated with chemoradiotherapy vs. 12.4 months if treated with radiotherapy alone; $\mathrm{HR}=1.645$, 95\% CI: $1.075-2.517 ; p=0.022)$ and radiotherapy dose $\geq 60 \mathrm{~Gy}$ (20.2 months if radiotherapy dose $\geq 60 \mathrm{~Gy} v s .10 .9$ months if radiotherapy dose $<60 \mathrm{~Gy}$; $\mathrm{HR}=1.642,95 \% \mathrm{CI}$ : $1.065-2.530 ; p=0.025$ ) were identified as independent prognostic factors of OS.

\section{Discussion}

There are still no standard therapeutic strategies for elderly patients with EC. It was previously reported that, at 70 years of age, life expectancy is $>10$ years (Arias, 2014). Therefore, provided there are no major comorbidities, elderly patients with EC may benefit from curative treatment. Several studies (Kawashima et al., 1998; Tougeron et al., 2008; Uno et al., 2004) indicated that an age of 70 years did not affect the frequency of adverse events, age was not a significant prognostic risk factor for EC patients treated with chemoradiotherapy, which were in accordance with the present study. In our study, it was shown that the median OS of elderly EC patients treated with radiotherapy or chemoradiotherapy was 17.9 months, which were not so far from the results published in the literature for younger counterparts. Several important clinical trials (Bedenne et al., 2007; Stahl et al., 2009) reported median OS ranged between 13.0 and 19.3 months for EC patients below the age of 75 years old. This emphasizes the fact that chemoradiotherapy in elderly patients is feasible and it may represent an important treatment modality for the elderly as well as for younger EC patients. These results are in line with previous findings (Rochigneux et al., 2014).

In the present study, we compared treatment outcomes between chemoradiotherapy and radiotherapy as well as radiotherapy dose $\geq 60 \mathrm{~Gy}$ and $<60 \mathrm{~Gy}$. We demonstrated that both chemoradiotherapy and radiotherapy dose $\geq 60 \mathrm{~Gy}$ improved OS without a significant increase in treatment-related mortalities or toxicities. Furthermore, in multivariate analysis, chemoradiotherapy and radiotherapy dose $\geq 60$ Gy were significant prognostic factors for improved OS. This lends support to the conclusion that the combined modality is superior to radiotherapy alone, as well as radiotherapy dose $\geq 60 \mathrm{~Gy}$ is superior to $<60 \mathrm{~Gy}$. Moreover, our results showed that radiotherapy dose 60Gy improved OS statistically significant compared with radiotherapy dose $<60 \mathrm{~Gy}$, while the difference between radiotherapy dose $60 \mathrm{~Gy}$ and $>60 \mathrm{~Gy}$ was not statistically significant. Our study also showed that cCRT improved OS significantly compared with sCRT and radiotherapy alone. These emphasizes the fact that radiotherapy dose $60 \mathrm{~Gy}$ is an effective treatment option for elderly EC patients while higher dose is not beneficial to improve OS, cCRT is superior to both sCRT and radiotherapy alone in terms of OS.

The National Comprehensive Cancer Network (NCCN) esophageal cancer guidelines recommend 50 or 50.4 Gy as standard radiotherapy dose for definitive chemoradiotherapy, which was based on the results of the RTOG trial 94-05 (Minsky et al., 2002). However, several studys showed that higher dose radiotherapy improved survival compared with standard dose radiotherapy (Suh et al., 2014), and the regimen of concurrent chemoradiotherapy with 60Gy radiotherapy in 30 fractions is widely used in Japan (Hironaka et al., 2003; Li et al., 2010; Suh et al., 2014). The dose of radiotherapy requires further investigation. Suh et al (Suh et al., 2014) compared treatment response to concurrent chemoradiotherapy using high-dose (>60Gy, median dose 63Gy, range 60 - 81Gy) versus standard-dose ( $<60 \mathrm{~Gy}$, median dose 54Gy, range 45 - 59.4Gy) radiotherapy in patients with stages II - III EC, it showed that high-dose radiotherapy of 60Gy or higher with concurrent chemotherapy improved locoregional control and progression-free survival without a significant increase of treatment-related toxicity. The conclusion agreed with the present study. Nevertheless, median OS for the high- and the standard-dose groups was 28 and 18 months, respectively, and The Japan Clinical Oncology Group (JCOG) 9906 trial (Li et al., 2010) showed that for stages II - III esophageal squamous cell carcinoma treated with 60Gy radiotherapy concurrent with chemotherapy of cisplatin and 5-FU the median OS was 29 months, which showed a better prognosis than the radiotherapy dose of 60Gy in the present study. The difference in median OS could be partially explained by excluding stages IV patients and patients were treated with concurrent 
chemoradiotherapy in these trials.

Several studies showed that chemoradiotherapy is an effective and safety treatment in elderly patients with EC (Nallapareddy et al., 2005; Tougeron et al., 2008; Uno et al., 2004; Xu et al., 2014), which were in accordance with the present study, while median OS for patients treated with concurrent chemoradiotherapy in the present study was 22.3 months, which showed a better prognosis than those published in the literature. A small prospective study (Kawashima et al., 1998) of 22 patients $>75$ years old treated by exclusive chemoradiotherapy (50Gy and weekly cisplatin), the median OS was 15 months. D Tougeron et al (Tougeron et al., 2008) reported that 109 EC patients over 70 years treated by exclusive chemoradiotherapy (50 - 55Gy and CDDP/5FU or CDDP/irinotecan) the median OS was 15.2 months. This might be partially explained by the higher RT dose used in the present study.

Concerning treatment tolerance, the difference in grade 3-4 toxicity between patients treated with chemoradiotherapy and radiotherapy alone as well as radiotherapy dose $\geq 60 \mathrm{~Gy}$ and $<60 \mathrm{~Gy}$ were not statistically significant. Febrile neutropenia and nutritional status are two challenges in elderly EC patients treated with chemoradiotherapy. Thus close monitoring of nutritional status and leucocyte of these patients is necessary. Essentially medical intervention such as using nutritional supplements or enteral nutrition to keep a sufficient calories intake or relying on GCSF to prevent neutropenia is useful.

Due to its retrospective nature, our analysis suffers from limitations. Firstly, the possibility of underestimating treatment-related toxicities. Secondly, unbalance of patient characteristics between the groups. Thirdly, a median follow-up of 16.97 months is rather short to precisely evaluate OS, as well as their prognostic factors. Fourthly, dosages and cycles of chemotherapy were various according to patients. However, despite these limitations, our results suggest that definitive concurrent chemoradiotherapy could be considered as a feasible and effective treatment in EC patients aged 70 and older, radiotherapy dose 60Gy is an effective treatment option compared with standard dose radiotherapy, while higher dose is not beneficial to improve survival.

\section{Acknowledgements}

This work was supported by the National Natural Science Foundation of China (No. 81372518).

\section{References}

Arias E. (2014). United states life tables, 2009. Natl Vital Stat Rep, 62, 1-63.

Bedenne L, Michel P, Bouche O, et al (2007). Chemoradiation followed by surgery compared with chemoradiation alone in squamous cancer of the esophagus: FFCD 9102. J Clin Oncol, 25, 1160-8.

Chen Z, Chen Q, Xia H, Lin J (2011). Green tea drinking habits and esophageal cancer in southern China: a case-control study. Asian Pac J Cancer Prev, 12, 229-33.

D'Journo XB, Thomas PA(2014). Current management of esophageal cancer. J Thorac Dis, 6, 253-64.

Hironaka S, Ohtsu A, Boku N, et al (2003). Nonrandomized comparison between definitive chemoradiotherapy and radical surgery in patients with $\mathrm{T}(2-3) \mathrm{N}$ (any) M (0) squamous cell carcinoma of the esophagus. Int J Radiat Oncol Biol Phys, 57, 425-33.

Igissinov N, Tereshkevich D, Moore MA, et al (2011). Age characteristics of incidences of prevalent cancers in the Aral Sea area of Kazakhstan. Asian Pac J Cancer Prev, 12, 2295-7.

Kawashima M, Ikeda H, Yorozu A, et al. (1998). Clinical features of esophageal cancer in the octogenarian treated by definitive radiotherapy: a multi-institutional retrospective survey. Jpn J Clin Oncol, 28, 301-7.

Li G, Hu W, Wang J, et al (2010). Phase II study of concurrent chemoradiation in combination with erlotinib for locally advanced esophageal carcinoma. Int J Radiat Oncol Biol Phys, 78, 1407-12.

Liu SZ, Wang B, Zhang F, et al (2013). Incidence, survival and prevalence of esophageal and gastric cancer in Linzhou city from 2003 to 2009. Asian Pac J Cancer Prev, 14, 6031-4.

Minsky BD, Pajak TF, Ginsberg RJ, et al (2002). INT 0123 (Radiation Therapy Oncology Group 94-05) phase III trial of combined-modality therapy for esophageal cancer: high-dose versus standard-dose radiation therapy. J Clin Oncol, 20, 1167-74.

Mirinezhad SK, Somi MH, Seyednezhad F, et al (2013). Survival in patients treated with definitive chemoradiotherapy for non-metastatic esophageal cancer in north- west iran. Asian Pac J Cancer Prev, 14, 1677-80.

Mirinezhad SK, Somi MH, Shirmohamadi M, et al (2013). Impact of postoperative chemoradiotherapy and chemoradiotherapy alone for esophageal cancer in NorthWest Iran. Asian Pac J Cancer Prev, 14, 3921-4.

Mountain CF, Dresler CM (1997). Regional lymph node classification for lung cancer staging. Chest, 111, 1718-23.

Nallapareddy S, Wilding GE, Yang G, Iyer R, Javle M (2005). Chemoradiation is a tolerable therapy for older adults with esophageal cancer. Anticancer Res, 25, 3055-60.

Rochigneux P, Resbeut M, Rousseau F, et al (2014). Radio (chemo) therapy in elderly patients with esophageal cancer: a feasible treatment with an outcome consistent with younger patients. Front Oncol, 4, 100.

Shen K, Huang XE, Lu YY, Wu XY, Liu J, Xiang J (2012). Phase II study of docetaxel (Aisu (R)) combined with three- dimensional conformal external beam radiotherapy in treating patients with inoperable esophageal cancer. Asian Pac J Cancer Prev, 13, 6523-6.

Stahl M, Walz M K, Stuschke M, et al (2009). Phase III comparison of preoperative chemotherapy compared with chemoradiotherapy in patients with locally advanced adenocarcinoma of the esophagogastric junction. J Clin Oncol, 27, 851-6.

Suh YG, Lee IJ, Koom WS, et al (2014). High-dose versus standard-dose radiotherapy with concurrent chemotherapy in stages II-III esophageal cancer. Jpn J Clin Oncol, 44, 534-40.

Tougeron D, Di Fiore F, Thureau S, et al (2008). 
Safety and outcome of definitive chemoradiotherapy in elderly patients with oesophageal cancer. Br J Cancer, 99, 1586-92.

Uno T, Isobe K, Kawakami H, et al (2004). Efficacy and toxicities of concurrent chemoradiation for elderly patients with esophageal cancer. Anticancer Res, 24, 2483-6.

Xu H Y, ZD, Zhou L, et al (2014). Safety and efficacy of radiation and chemoradiation in patients over 70 years old with inoperable esophageal squamous cell carcinoma. Oncol Lett, 7, 260-6.

Yuan P, Chen TH, Chen ZW, Lin XQ. (2014). Calculation of life-time death probability due malignant tumors based on a sampling survey area in China. Asian Pac J Cancer Prev, 15, 4307-9.

Zhang Y. (2013). Epidemiology of esophageal cancer. World J Gastroenterol, 19, 5598-606. 